\title{
The Field Emission Properties of Graphene Aggregates Films Deposited on Fe-Cr-Ni alloy Substrates
}

\author{
Zhanling Lu, ${ }^{1}$ Wanjie Wang, ${ }^{1}$ Xiaotian Ma, ${ }^{1}$ Ning Yao, ${ }^{2}$ Lan Zhang, ${ }^{3}$ and Binglin Zhang ${ }^{3}$ \\ ${ }^{1}$ School of Materials Science and Engineering, Zhengzhou University, Zhengzhou 450001, China \\ ${ }^{2}$ Materials Physics Laboratory of Education Ministry of China, Zhengzhou University, Zhengzhou 450052, China \\ ${ }^{3}$ Department of Engineering Mechanics, Zhengzhou University, Zhengzhou 450001, China
}

Correspondence should be addressed to Zhanling Lu, zllu@zzu.edu.cn

Received 31 May 2010; Accepted 21 June 2010

Academic Editor: Rakesh Joshi

Copyright ( $) 2010$ Zhanling Lu et al. This is an open access article distributed under the Creative Commons Attribution License, which permits unrestricted use, distribution, and reproduction in any medium, provided the original work is properly cited.

\begin{abstract}
The graphene aggregates films were fabricated directly on $\mathrm{Fe}-\mathrm{Cr}-\mathrm{Ni}$ alloy substrates by microwave plasma chemical vapor deposition system (MPCVD). The source gas was a mixture of $\mathrm{H}_{2}$ and $\mathrm{CH}_{4}$ with flow rates of $100 \mathrm{sccm}$ and $12 \mathrm{sccm}$, respectively. The micro- and nanostructures of the samples were characterized by Raman scattering spectroscopy, field emission scanning electron microscopy (SEM), and transparent electron microscopy (TEM). The field emission properties of the films were measured using a diode structure in a vacuum chamber. The turn-on field was about $1.0 \mathrm{~V} / \mu \mathrm{m}$. The current density of $2.1 \mathrm{~mA} / \mathrm{cm}^{2}$ at electric field of $2.4 \mathrm{~V} / \mu \mathrm{m}$ was obtained.
\end{abstract}

\section{Introduction}

Graphene has grabbed appreciable attention due to its exceptional electronic and optoelectronic properties [1]. One of the potential applications of graphene is in field emission (FE) displays. Malesevic et al. synthesized vertically aligned few-layer graphenes (FLGSs) using plasma-enhanced chemical vapor deposition (PECVD) on titanium substrate, and turn-on field of the field emission from the graphene layer was as low as $1 \mathrm{~V} / \mu \mathrm{m}$ [2]. Qi et al. prepared FLGSs by radio-frequency $\mathrm{PECVD}$ on $\mathrm{Si}(100)$ substrates without any catalyst, and turn-on field of its emission was $3.91 \mathrm{~V} / \mu \mathrm{m}$ [3]. We previously reported that nanocrystalline graphitic films on ceramic substrates by MPCVD using $\mathrm{Fe}-\mathrm{Ni}$-Cr layer as catalyst were deposited, the turn-on field of $1.26 \mathrm{~V} / \mu \mathrm{m}$ was obtained [4].

In this paper, we present the field emission characteristics of the graphene aggregates films, which were synthesized by MPCVD directly on stainless steel substrate without any catalyst. The structure of the films was also studied.

\section{Experiment}

The Fe-Cr-Ni alloy substrates were polished with abrasive paper of W7 (particle size was about $15 \mu \mathrm{m}$ ) and then ultrasonicaly cleaned with deionized water and acetone. The graphene films were deposited directly on the substrates without any catalyst by MPCVD. The source gas was a mixture of $\mathrm{H}_{2}$ and $\mathrm{CH}_{4}$ with flow rates of $100 \mathrm{sccm}$ and $12 \mathrm{sccm}$, respectively. During the deposition, the total pressure of $6.0 \mathrm{KPa}$, substrate temperature of $700^{\circ} \mathrm{C}$, and microwave power of $1600 \mathrm{~W}$ were kept for 10 mins.

The structure of deposited films were analyzed by Raman scattering, SEM, and TEM. The field emission properties were measured using a diode structure, which was placed in a vacuum chamber. The deposited film with area of $0.2 \mathrm{~cm}^{2}$ was used as a cathode. The anode of an indium tin oxide (ITO-) coated glass was separated from the cathode by a mica spacer with thickness of $600 \mu \mathrm{m}$. The base pressure in the vacuum chamber was maintained below $1 \times 10^{-4} \mathrm{~Pa}$ during the measurements. The voltage was stepped up from 0 to $2500 \mathrm{~V}$.

\section{Results and Discussion}

The Raman spectrum of the sample was shown in Figure 1. The G- band appearing at $1583 \mathrm{~cm}^{-1}$ originates from in-plane vibration of $\mathrm{sp}^{2}$ carbon atoms and is a doubly degenerate (TO and LO) phonon mode ( $\mathrm{E}_{2 \mathrm{~g}}$ symmetry) at 


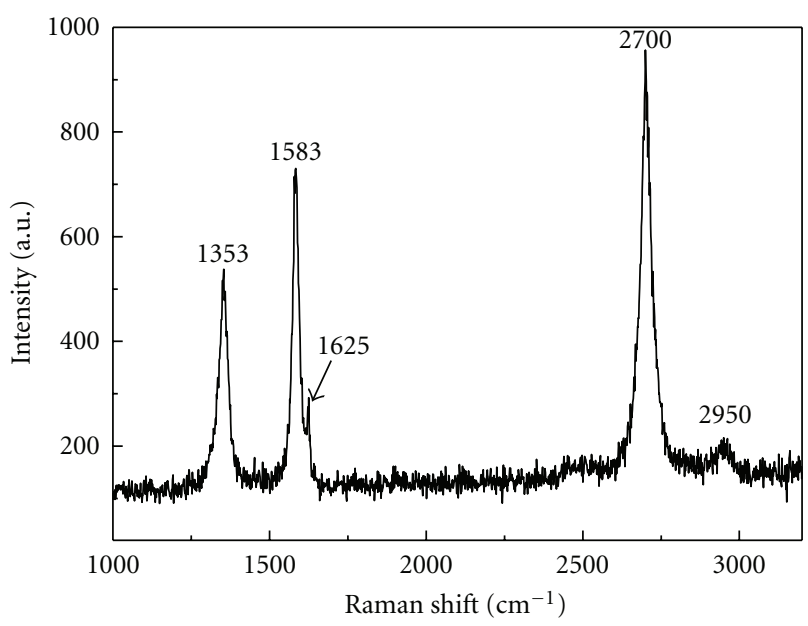

FIGURE 1: Raman shift of the as-grown sample.

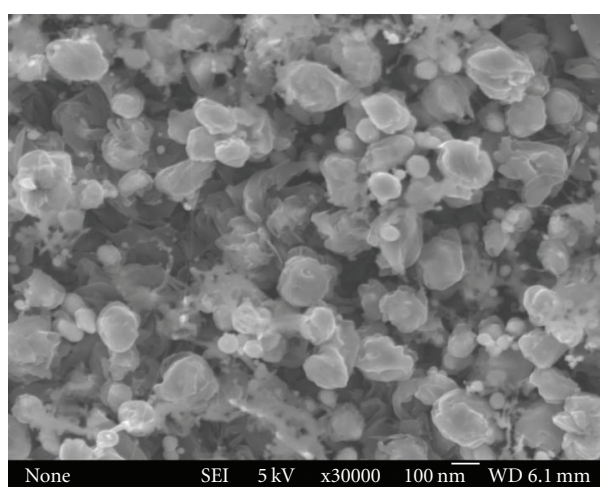

(a)

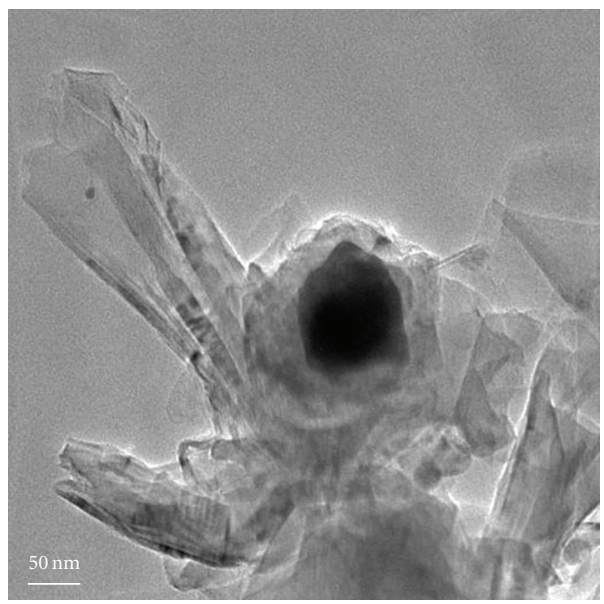

(c)

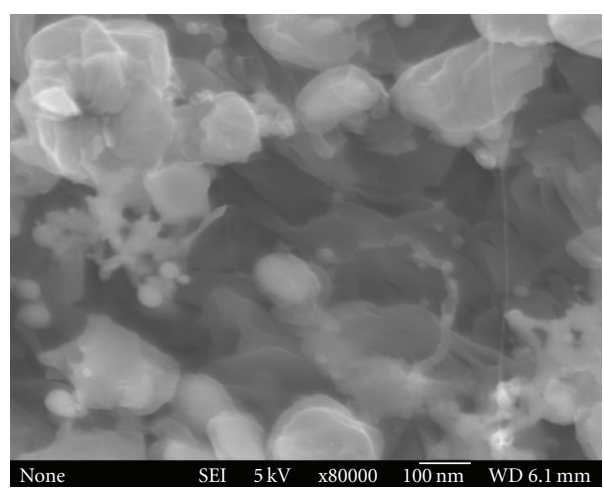

(b)

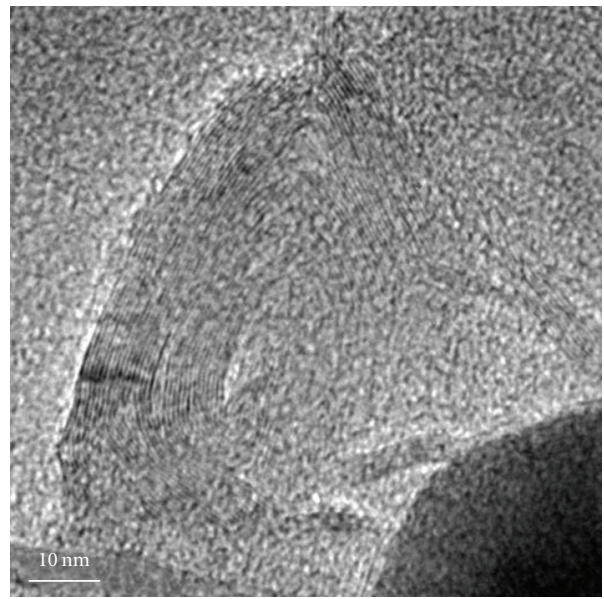

(d)

FIGURE 2: SEM and TEM images of the sample; (a) top-view SEM image of as-grown sample, (b) SEM of an enlarged image, (c) TEM image of agglomerated graphene sheets, and (d) HRTEM image of graphene sheet.

the Brillouin zone center. The band located at $1353 \mathrm{~cm}^{-1}$ corresponds to the $\mathrm{D}$ band of graphitic carbon species, which is a disorder-activated Raman mode and is associated with the TO branch near the K-point. The $2 \mathrm{D}$ band at $2700 \mathrm{~cm}^{-1}$ originates from a two-phonon double resonance Raman process, and it is closely related to the band structure of graphene layers and is to be used to confirm the presence of graphene $[5,6]$. The band at $1620 \mathrm{~cm}^{-1}$, termed as $\mathrm{D}^{\prime}$, which arises from phonon scattering at the crystal-lattice defects. The band at $2950 \mathrm{~cm}^{-1}$ originates from $\mathrm{D}+\mathrm{G}$ mode 


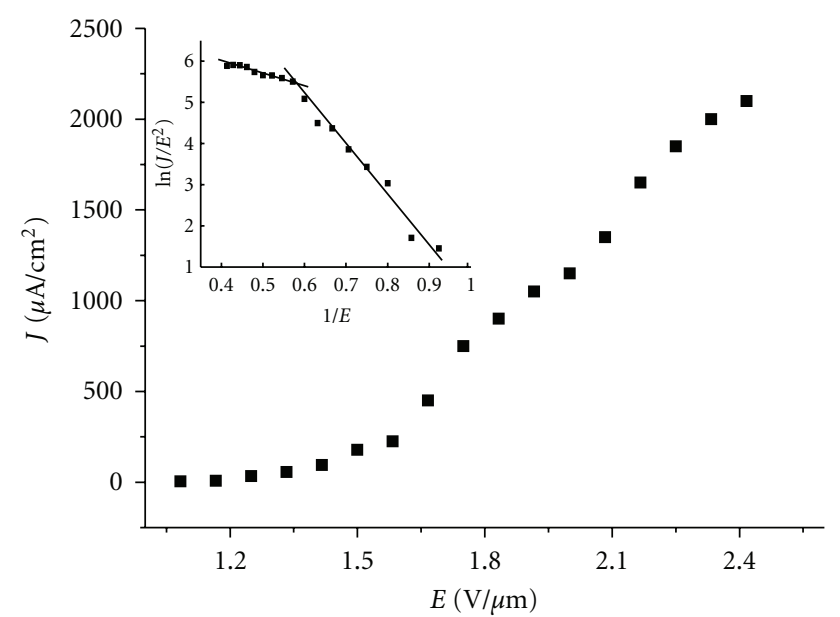

Figure 3: I-V curve and its F-N plot of the sample.

[7]. The Raman spectrum of the sample indicated that the deposited film was graphene film.

The morphology of the graphene film was shown in Figure 2. Figures 2(a) and 2(b) showed the top-view SEM images of as-grown sample, and it seemed to be consisted of petal-like clusters of graphene sheets. Such kind of film was named graphene aggregates film. The sizes of the clusters were about 100-300 nanometers. In order to further examine and confirm the nanostructure of the sample, TEM measurements were performed. To prepare TEM samples, the sample was ultrasonicaly treated in alcohol directly, then dropped onto holey grids. Figure 2(c) revealed the TEM image of agglomerated graphene sheets around an amorphous carbon particle, which may hint that the growth of the graphene sheets probably originated from amorphous carbon structure. In the experimental condition, the detailed growth mechanism of the agglomerated graphene needs further study. The layered structure shown in HRTEM image (Figure 2(d)) confirmed the structrue of graphene. It also revealed the defects such as wrinkles and edge loops in graphene sheet.

The field emission characteristics of the graphene aggregates film were tested. The turn-on field of about $1.0 \mathrm{~V} / \mu \mathrm{m}$ and the current density of $2.1 \mathrm{~mA} / \mathrm{cm}^{2}$ at electric field of $2.4 \mathrm{~V} / \mu \mathrm{m}$ were obtained. Figure 3 showed the current versus voltage $(I-V)$ curve of the field emission, and the corresponding Fower-Nordheim (F-N) plot was inserted in Figure 3, which characterized the tunnel effect of the field emission from the graphene aggregates film. The F-N plot exhibited two different slopes at low-field region and highfield region. The current saturation of the emission and the slope deviation of the F-N plot at high-field region were observed in Figure 3. The same phenomena were observed for carbon nanotubes emitter, reported by Choi et al. [8]. In the present case, we suggested that the current saturation and F-N plot deviation were attributed to the same reason, which was adsorbate desorption effect, especially hydrogenadsorbate desorption during the emission measurement. We had also reported the hydrogen desorption effect during the emission measurement for amorphous carbon emitter [9]. During the graphene film deposition by CVD method, hydrogen atoms were adsorbed on the surface and edges of graphene aggregates to form the localized states near the Fermi level, which were responsible for the emission. During the emission measurement, the hydrogen adsorbates gradually desorbed, and the localized states disappeared, which probably led to the current saturation and F-N plot deviation phenomena.

The above measured results indicate that the graphene aggregates film was a good material for field electron emitter. The good field emission properties would be attributed to two possible mechanisms. (1) The graphene sheets were extending to a height of several hundreds of nanometers vertically aligned to their surfaces along the applied electric field, and the field sites of which would cause a large-field enhancement factor [10]. (2) the defects of the graphene sheet lowered electron affinity that provided a low-energy barrier and enhanced electron emission. Filleter et al. reported that energy barrier of single-layer graphene grown epitaxially on $6 \mathrm{H}-\mathrm{SiC}(0001)$ had been determined to be $135 \pm 9 \mathrm{meV}$ by means of the Kelvin probe force microscopy [11], which was much lower than that of $4.5 \mathrm{eV}$ for graphite.

\section{Conclusion}

In summary, we have demonstrated that the graphene film can be directly grown on stainless steel surface employing methane as the source gas in about a 10-min growth time. The growth temperature was about $700^{\circ} \mathrm{C}$. The graphene aggregates emitter has a very low turn-on field of only about $1 \mathrm{~V} / \mu \mathrm{m}$ and an emission current density of $2.1 \mathrm{~mA} / \mathrm{cm}^{2}$ at an applied field of $2.4 \mathrm{~V} / \mu \mathrm{m}$. This promising field emission performance can be attributed to the electric field enhancement, and the defects induced low-work function of the graphene aggregates film.

\section{References}

[1] W. Choi, I. Lahiri, R. Seelaboyina, and Y. S. Kang, "Synthesis of graphene and its applications: a review," Critical Reviews in Solid State and Materials Sciences, vol. 35, no. 1, pp. 52-71, 2010.

[2] A. Malesevic, R. Kemps, A. Vanhulsel, M. P. Chowdhury, A. Volodin, and C. V. Haesendonck, "Field emission from vertically aligned few-layer graphene," Journal of Applied Physics, vol. 104, no. 8, Article ID 084301, 5 pages, 2008.

[3] J. L. Qi, X. Wang, W. T. Zheng, H. W. Tian, C. Q. Hu, and Y. S. Peng, "Ar plasma treatment on few layer graphene sheets for enhancing their field emission properties," Journal of Physics $D$, vol. 43, no. 5, Article ID 055302, 6 pages, 2010.

[4] J. Deng, L. Zhang, B. Zhang, and N. Yao, "The structure and field emission enhancement properties of nano-structured flower-like graphitic films," Thin Solid Films, vol. 516, no. 21, pp. 7685-7688, 2008.

[5] Z. H. Ni, Y. Y. Wang, T. Yu, and Z. X. Shen, "Raman spectroscopy and imaging of graphene," Nano Research, vol. 1, no. 4,pp. 273-291, 2008. 
[6] L. M. Malard, M. A. Pimenta, G. Dresselhaus, and M. S. Dresselhaus, "Raman spectroscopy in graphene," Physics Reports, vol. 473, no. 5-6, pp. 51-87, 2009.

[7] C. N. R. Rao, A. K. Sood, K. S. Subrahmanyam, and A. Govindaraj, "Graphene: the new two-dimensional nanomaterial," Angewandte Chemie-International Edition, vol. 48, no. 42, pp. 7752-7777, 2009.

[8] Y. C. Choi, Y. M. Shin, D. J. Bae, S. C. Lim, Y. H. Lee, and B. S. Lee, "Patterned growth and field emission properties of verticallyaligned carbon nanotubes," Diamond and Related Materials, vol. 10, no. 8, pp. 1457-1464, 2001.

[9] Z.-L. Lu, C.-Q. Wang, Y. Jia, B.-L. Zhang, and N. Yao, "Electron emission degradation of nano-structured $\mathrm{sp}^{2}$-bonded amorphous carbon films," Chinese Physics, vol. 16, no. 3, pp. 843-847, 2007.

[10] A. Y. Babenko, A. T. Dideykin, and E. D. Eidelman, "Graphene ladder: a model of field emission center on the surface of loose nanocarbon materials," Physics of the Solid State, vol. 51, no. 2, pp. 435-439, 2009.

[11] T. Filleter, K. V. Emtsev, T. Seyller, and R. Bennewitz, "Local work function measurements of epitaxial graphene," Applied Physics Letters, vol. 93, no. 13, Article ID 133117, 2008. 

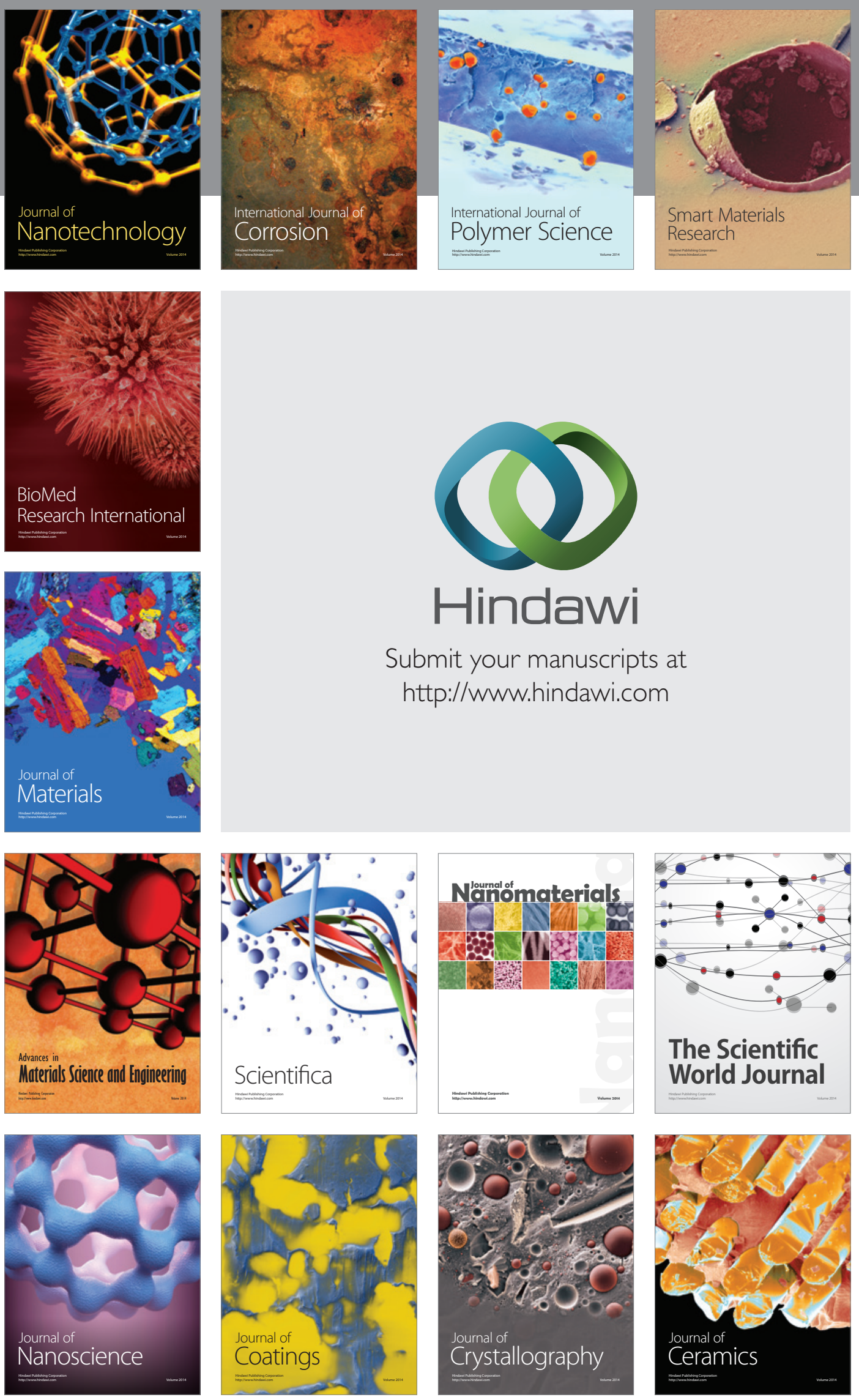

The Scientific World Journal

Submit your manuscripts at

http://www.hindawi.com

\section{World Journal}

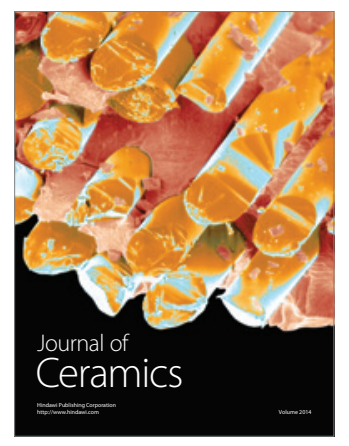

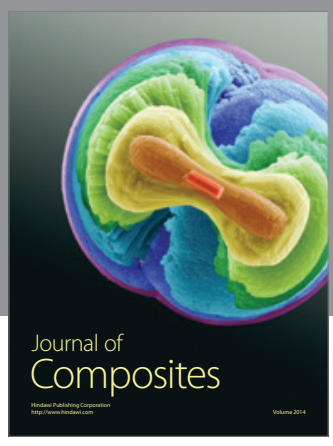
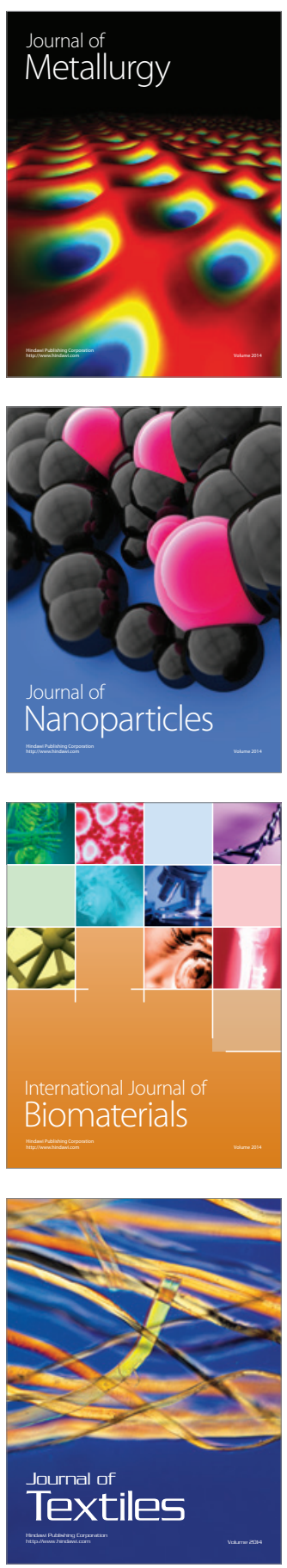